\title{
Primary pelvic retroperitoneal ancient schwannoma-a rare diagnosis of pelvic complex cystic lesion
}

\author{
TS Chan *, T Wong, NY Pan
}

Hong Kong Med J 2019;25:160-1.e1-3

http://doi.org/10.12809/hkmj187188

A 42-year-old woman was admitted through our emergency department for subacute onset of lower abdominal pain in September 2014. Bedside ultrasound by a gynaecology specialist showed a left adnexal cyst with fluid interface. Magnetic resonance imaging of the pelvis showed a large well-defined multiloculated cystic lesion measuring $9.2 \mathrm{~cm}$ (width) $\times 8.5 \mathrm{~cm}($ depth $) \times 8.9 \mathrm{~cm}$ (height) with thick $\mathrm{T} 1$ and $\mathrm{T} 2$ hypointense enhancing wall and septa at the left side of the pelvis (Fig 1). An internal slightly
T1 hyperintense component with fluid-fluid level was suspected to be proteinaceous or haemorrhagic content. A 1.6- $\mathrm{cm}$ non-enhancing mural nodule was noted at the posterior aspect. Partial bicornuate uterus was noted. Bilateral ovaries were normal in size with small cysts (Fig 2). Laparoscopy was done, with excision of the lesion. Intra-operative frozen section showed a benign spindle cell tumour. Final histopathological evaluation revealed a retroperitoneal schwannoma.
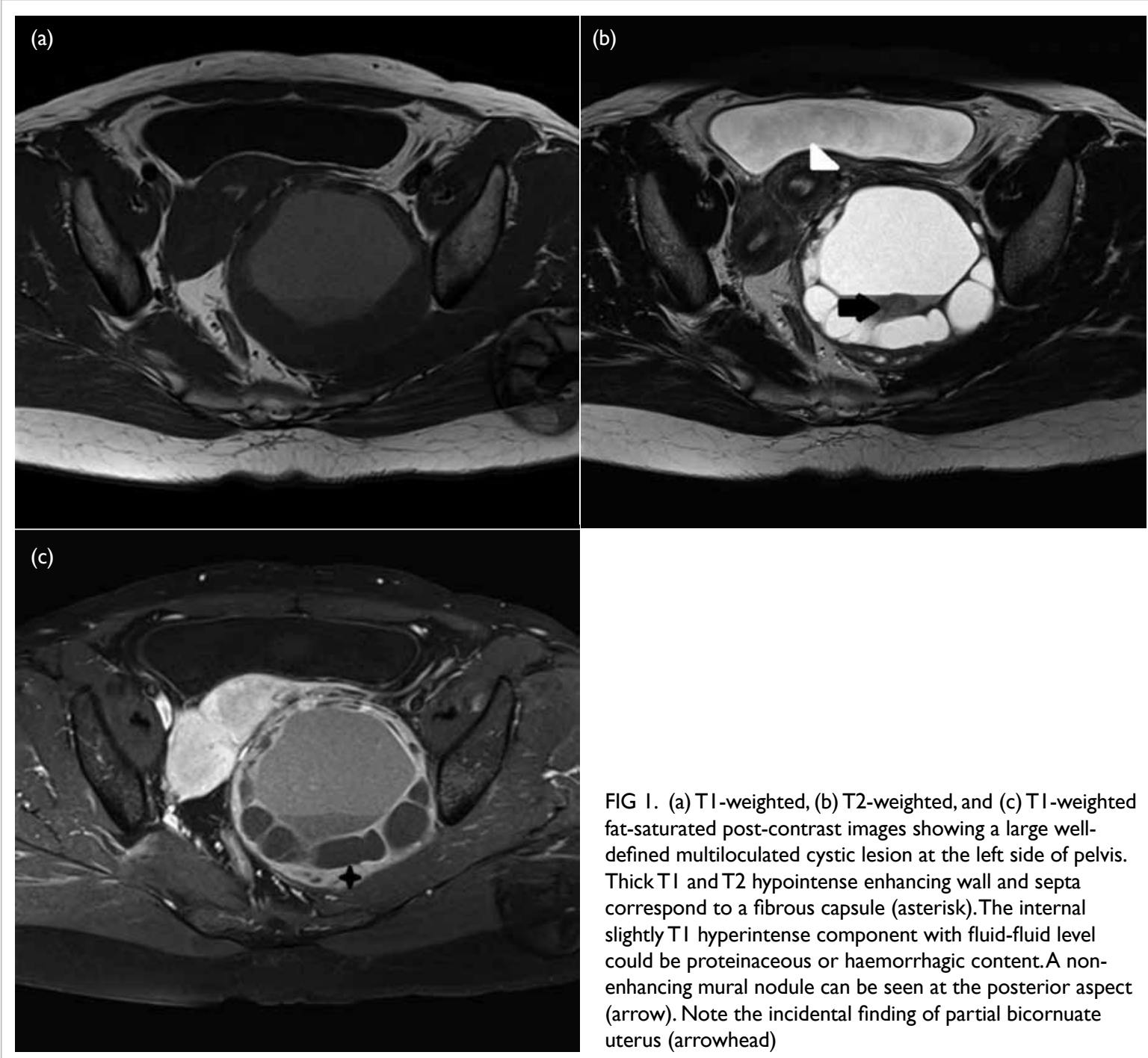

FIG I. (a) TI-weighted, (b) T2-weighted, and (c) TI-weighted fat-saturated post-contrast images showing a large welldefined multiloculated cystic lesion at the left side of pelvis. Thick TI and T2 hypointense enhancing wall and septa correspond to a fibrous capsule (asterisk). The internal slightly TI hyperintense component with fluid-fluid level could be proteinaceous or haemorrhagic content. A nonenhancing mural nodule can be seen at the posterior aspect (arrow). Note the incidental finding of partial bicornuate uterus (arrowhead) 


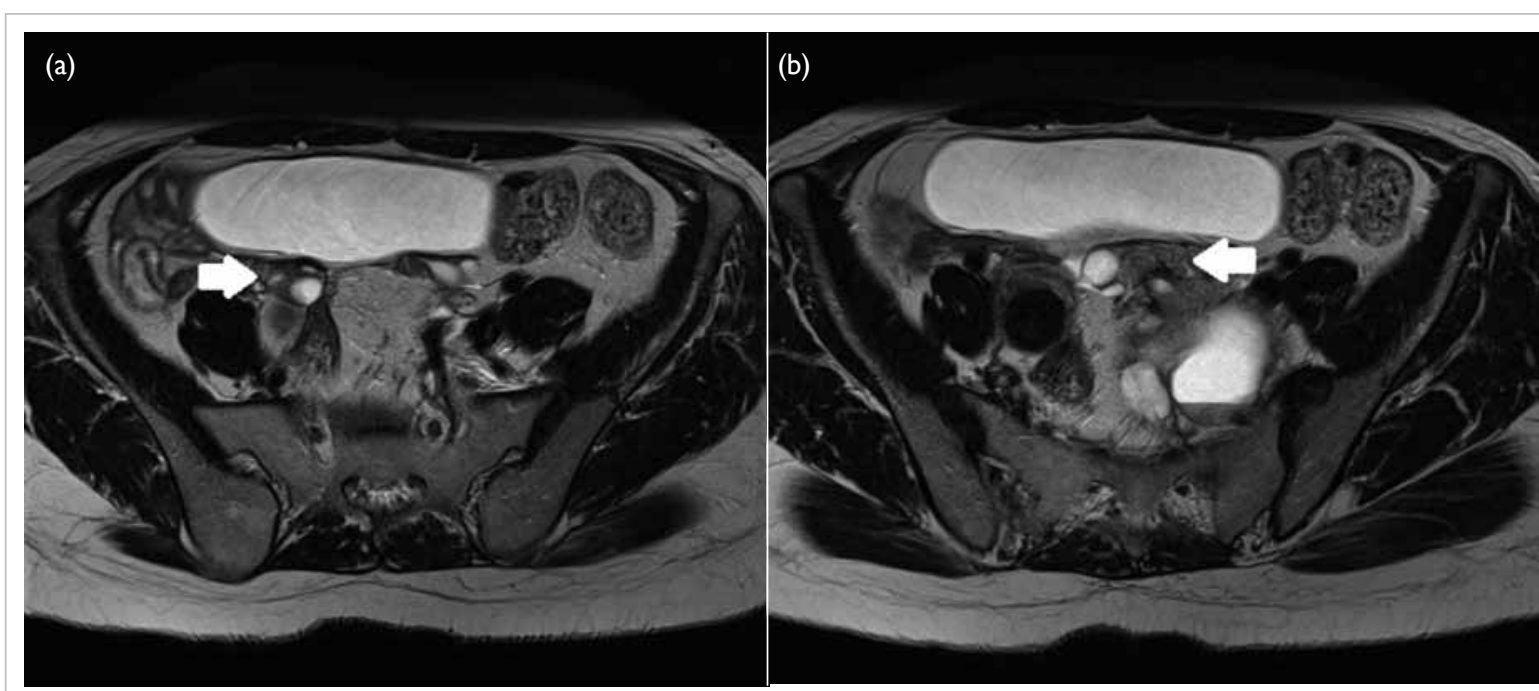

FIG 2. T2-weighted images showing (a) right and (b) left ovaries (arrows), which are normal in size and with small cysts

The majority of cystic pelvic masses originate from the ovary. Mimics of ovarian cystic masses have a wide variety of diagnoses. It is important to understand the relationship of a mass with its anatomic location, identify normal ovaries at imaging, and correlate imaging findings with the patient's clinical history to avoid misdiagnosis.

Retroperitoneal schwannoma is a rare tumour and is difficult to diagnose, accounting for only $6 \%$ of retroperitoneal neoplasms. A retroperitoneal schwannoma is usually located in the paravertebral space or pre-sacral pelvic retroperitoneum. ${ }^{1}$ It usually occurs in young to middle-aged adults, and women are affected twice as often as men. ${ }^{1}$ The patient is usually asymptomatic, or complains of a wide variety of non-specific symptoms when the tumour is large in size. ${ }^{2}$ Malignant transformation is rare. ${ }^{1}$ On magnetic resonance images, a schwannoma appears as a well-defined mass with hypo- or iso-intensity on T1-weighted images and with hyperintensity on T2weighted images. The nerve of origin is often difficult to identify. It is not unusual for a schwannoma to display cystic changes. However, prominent cystic changes are uncommon and point to ancient schwannoma, a rare variant of schwannoma that is characterised by degeneration and decreased cellularity. ${ }^{3}$ On magnetic resonance images, ancient schwannoma appears as a well-defined, complex cystic mass with a variable enhancement pattern. Thick T1 and T2 hypointense enhancing wall and septa correspond to a fibrous capsule, consisting of epineurium and residual nerve fibres. ${ }^{4}$

Identification of the nerve adjacent to or along the tumour is useful for differentiating ancient schwannomas from other complex cystic lesions, such as serous or mucinous cystadenocarcinoma, abscess, necrotic soft-tissue sarcoma, or necrotic metastatic lymphadenopathy. ${ }^{5}$

In the present case, the patient did not complain of any neurological symptoms at presentation. The presence of normal ovaries and fibrous capsule indicated a preoperative diagnosis of ancient schwannoma. This case illustrates the importance of considering this uncommon diagnosis when a pelvic complex cystic lesion is detected in imaging, and seeking specific imaging features (such as fibrous capsule and close relationship to the nerve) to confirm or exclude this diagnosis. This would facilitate surgical planning and minimise the risk of complications such as major neurological deficit.

\section{Author contributions}

All authors contributed to the concept or design, acquisition of data, analysis or interpretation of data, drafting of the manuscript, and critical revision for important intellectual content. All authors had full access to the data, contributed to the study, approved the final version for publication, and take responsibility for its accuracy and integrity.

\section{Conflicts of interest}

All authors have disclosed no conflicts of interest.

\section{Funding/support}

This research received no specific grant from any funding agency in the public, commercial, or not-for-profit sectors.

TS Chan *, MB, BS, FRCR

T Wong, FRCR, FHKAM (Radiology)

NY Pan, FRCR, FHKAM (Radiology)

Department of Radiology, Princess Margaret Hospital, Kwai Chung, Hong Kong

*Corresponding author: drsunchan@gmail.com 


\section{References}

1. Rha SE, Byun JY, Jung SE, Chun HJ, Lee HG, Lee JM. Neurogenic tumors in the abdomen: tumor types and imaging characteristics. Radiographics 2003;23:29-43.

2. Kim SH, Choi BI, Han MC, Kim YI. Retroperitoneal neurilemoma: CT and MR findings. AJR Am J Roentgenol 1992;159:1023-6.
3. Dahl I. Ancient neurilemmoma (schwannoma). Acta Pathol Microbiol Scand A 1977;85:812-8.

4. Takeuchi M, Matsuzaki K, Nishitani H, Uehara H. Ancient schwannoma of the female pelvis. Abdom Imaging 2008;33:247-52.

5. Isobe K, Shimizu T, Akahane T, Kato H. Imaging of ancient schwannoma. AJR Am J Roentgenol 2004;183:331-6. 